\title{
N73 29961
}

\section{NASA TECHNICAL}

NASA TM X- 68276

\section{MEMORANDUM}

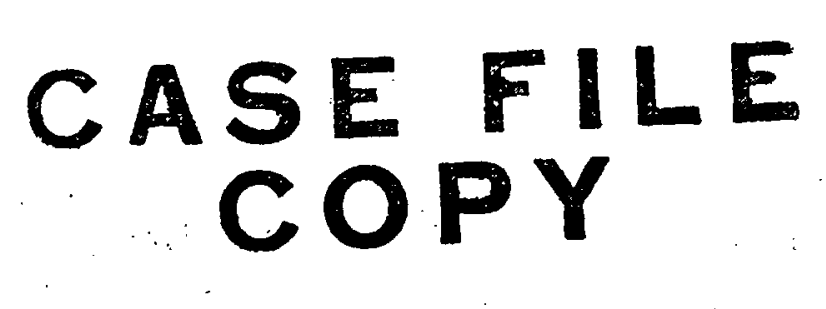

CONFORMAL MAPPING TECHNIQUE FOR TWO-DIMENSIONAL POROUS MEDIA AND JET IMPINGEMENT HEAT TRANSFER

by Robert Siegel

Lewis Research Center

Cleveland, Ohio 44135

TECHNICAL PAPER proposed for presentation at the Fifth International Heat Transfer Conference Tokyo, Japan, September 3-7, 1974 
CONFORMAL MAPPING TECHNIQUE FOR TWO-DIMENSIONAL POROUS MEDTA AND JET IMPINGEMENT HEAT TRANSFER

\author{
Robert Siegel \\ Lewis Research Center \\ National Aeronautics and Space Administration \\ Cleveland, Ohio U.S.A.
}

Abstract

Transpiration cooling and liquid metals both provide highly effective heat transfer. Using Darcy's law in porous media, and the inviscid approximation for liquid metals, the local fluid velocity in these flows equals the gradient of a potential. The energy equation and flow region are simplified when transformed into potential plane coordinates. In the se coordinates the present problems are reduced to heat conduction solutions which are mapped into the physical geometry. Results are obtained for a porous region with simultaneously prescribed surface temperature and heat flux, heat transfer in a two-dimensional porous bed, and heat transfer for two liquid metal slot jets impinging on a heated plate.

\section{NOMENCLATURE}

b : Characteristic dimension; half width of jet or coolant slot, m

$c_{p} \quad$ : specific heat of fluid at constant pressure, $\mathrm{kcal} / \mathrm{kg} \mathrm{K}$

$F$ : Volume flow rate per unit depth, $\mathrm{m}^{2} / \mathrm{s}$

$h_{1} h_{2}$ : Dimensionless jet widths, Fig. $6(\mathrm{a})$

$\mathrm{K}$ : Thermal conductivity of porous matrix $\mathrm{k}_{\mathrm{m}}$, or liquid metal $\mathrm{k}$, $\mathrm{kcal} / \mathrm{m} \mathrm{s} \mathrm{K}$

$\hat{n} \quad$ : Outward unit normal vector

Pe : Peclet number, $F / \alpha$

$\mathrm{p} \quad:$ Static pressure, $\mathrm{N} / \mathrm{m}^{2}$

Qtot : Total amount of heat transferred, kcal/s

$\mathrm{q}:$ Heat flux, $\mathrm{kcal} / \mathrm{s} \mathrm{m}^{2}$

$\mathrm{S} \quad$ : Surface; coolant exit from porous medium; half-spacing between jets, $m$

T: : Dimensionless temperature, $\left(t-t_{\infty}\right) \mathrm{k}_{\mathrm{m}} /$ $\mathrm{q}_{\mathrm{g}} \mathrm{2b}$ for porous region with free surface, $\left(t-t_{\infty}\right) k / q_{2} 2 b$ for jet, $\left(t-t_{\infty}\right) /\left(t_{w, r}-t_{\infty}\right)$ for porous bed

$t \quad:$ Temperature, $\mathbb{K}, r$

$\begin{array}{ll}\mathrm{u}, v & : \mathrm{x} \text { and } \mathrm{y} \text { velocity components, } \mathrm{m} / \mathrm{s} \\ \overrightarrow{\mathrm{u}} & : \text { Vector velocity, } \mathrm{m} / \mathrm{s} ; \overrightarrow{\mathrm{U}}=\overrightarrow{\mathrm{u}} /(\mathrm{F} / 2 \mathrm{~b})\end{array}$

$\mathrm{X}, \mathrm{Y} \quad$ : Dimensionless coordinates, $\mathrm{x} / 2 \mathrm{~b}, \mathrm{y} / 2 \mathrm{~b}$

$\mathrm{X}_{\mathrm{D}} \quad$ : Jet stagnation point

$x, y$ : Rectangular coordinates, m

a : Thermal diffusivity, $\mathrm{K} / \rho \mathrm{c}_{\mathrm{p}}, \mathrm{m}^{2} / \mathrm{s}$

$k \quad:$ Permeability of porous medium, $\mathrm{m}^{2}$

$\mu \quad$ : Fluid viscosity, $\mathrm{N} \mathrm{s} / \mathrm{m}^{2}$

$\rho \quad$ : Fluid density, $\mathrm{kg} / \mathrm{m}^{3}$

$\Phi \quad$ : Dimensionless potential, $\varphi / F ; \Phi_{S}=\varphi_{S} / F$

$\varphi \quad$ : Velocity potential, for porous medium $\varphi_{m^{2} / s}=\left(p_{0}-p\right) k / \mu ; \varphi_{s}=\left(p_{0}-p_{s}\right) k / \mu$,

$\psi \quad:$ Streom function, $\mathrm{m}^{2} / \mathrm{s}$; dimensionless

$\Psi=\Psi / F$

$\tilde{\nabla} \quad$ : Dimensionless gradient, $\hat{i}(\partial / \partial X)+\hat{j}(\partial / \partial Y)$

Subscripts

r : Reference value

s : : On surface where coolant exdts from

w : Wall

$\infty \quad:$ : At reservoir condition

$0 \quad: \quad$ On surface where coolant enters porous medium

1,2 : At wall 1 or 2 .

\section{INTRODUCTION}

In connection with advanced power producing devices, the heat transfer engineer must contend with high heat fluxes arising from increasing temperatures of working fluids to raise thermal efficiency, or from high operating temperatures required in devices such as fusion reactors. Two means of effective cooling are transpiration-cooled porous walls and liquid metal coolants. A comon feature of these flows is that locally the fluid velocity is equal to the gradient of a velocity potential. This is true for Darcy flow in porous media, and for the inviscid flow approximation in liquid-metal heat transfer analyses. As a consequence the energy equation acquires a simplified form when transformed into a "natural" system of potential and streamline coordinates. For the present problems the solutions in the potential plane are reduced to heat conduction solutions which are mapped into the physical system.

The use of the potential plane was developed for two- and three-dimensional porous cooling in [1] and [2], and for liquid metal heat transfer for flow across cylinders in [3]. The technique is further developed here to obtain: (1) the shape of a two-dimensional porous region to provide a desired surface temperature for a specified surface heat flux, (2) the heat transfer for a two-dimensional porous bed with specified boundary temperatures, and (3) the temperature distribution on a uniformly heated plate cooled by impinging liquid metal jets.

\section{ANALYSIS}

Figure 1 shows a two-dimensional porous region with coolant supplied through a slot. The upper surface receives a uniform heat flux and is to be maintained at a specified temperature. The shape of the surface is to be found to meet these restrictions. The geometry is in dimensionless form in Fig. 4(a) and, as will be discussed, maps into potential plane coordinates as show in Fig. 4(b). Figure 2 shows a porous region between solid walls $S_{1}$ and $S_{2}$ with specified temperature distributions. It is desired to obtain the total heat transferred to the flow. Figure 5 shows the dimensionless form and the mapping into the potential plane. The third situation analyzed is the impingement heat transfer of the parallel liquid metal. slot jets in Fig. 3. The dimensionless form and mapping into the potential plane are in Fig. 6.

\section{Governing Equations}

The solutions obtained here are for constant prop- 


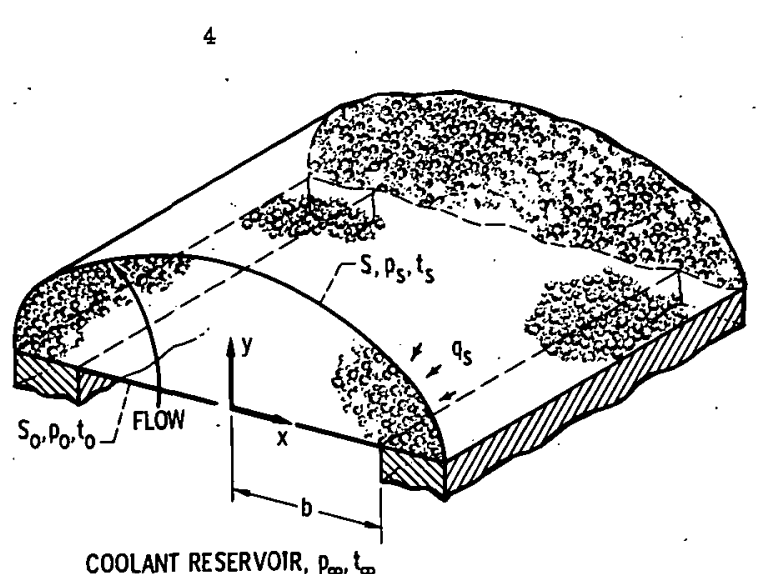

Fig. 1 POROUS LEADING EDGE REGION WITH SPECIFIED UNIFORM HEAT FLUX AND TEMPERATURE AT SURFACE

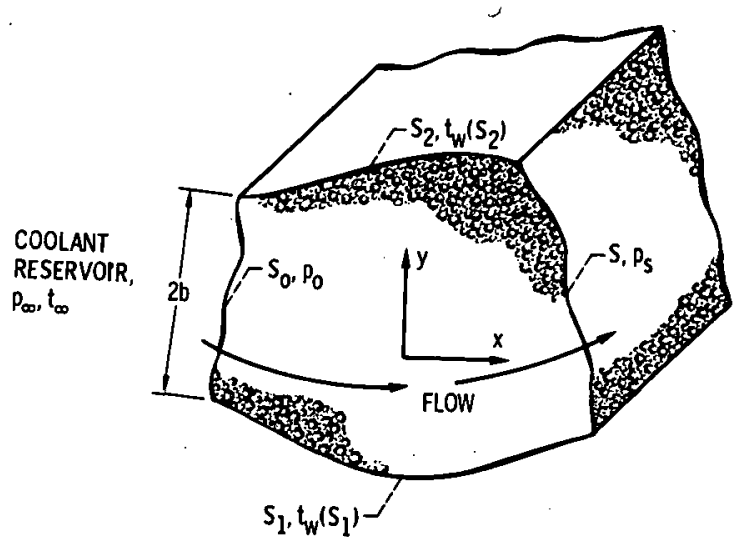

Fig. 2 POROUS BED WITH NONPOROUS SIDE WALLS MAINTAINED AT SPECIFIED TEMPERATURES

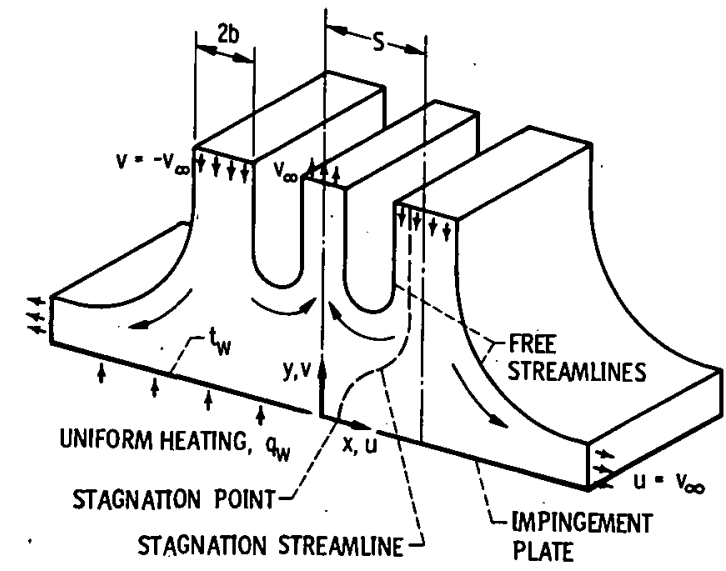

Fig. 3 PARALLEL SLOT JETS IIMPINGING AGAINST A UNIFORMLY HEATED . RLATE

erties. For heat transfer in a porous medium it is assumed that the coolant and porous matrix are in sufficiently good thermal contact that locally. they are at a cormon temperature. With these limitations the heat transfer in the porous medium or jet. is governed by the continuity and energy equations,

$\nabla \cdot \overrightarrow{\mathrm{u}}=0$

$K \nabla^{2} t-\rho C_{p} \vec{u} \cdot \nabla t=0$

In the porous medium the velocity is governed by Darcy's equation,

$\overrightarrow{\mathrm{u}}=-(\kappa / \mu) \nabla \mathrm{p}$

while in the liquid metal flow (assumed irrotational and inviscid) the velocity can be written as the gradient of a potential

$\overrightarrow{\mathrm{u}}=\nabla \varphi$

Define a potential $\varphi=\left(p_{0}-p\right) k / \mu$ and Eq. (3) becomes the same as Eq. (4). Thus the continuity, energy and flow equations have the same form for both the porous media and jet flows.

\section{Boundary Conditions}

The thermal boundary conditions are discussed in relation to each problem so only the pressure boundary conditions are given now. The static pressure drop as the fluid accelerates from the reservoir to the coolant inlet is usually small compared_with the pressure drop in the porous medium. Hence, along $\mathrm{S}_{0}$ in Figs. 1 and 2

$\mathrm{p}=\mathrm{p}_{0}=\mathrm{p}_{\infty}=$ constant $\mathrm{x}, \mathrm{y}$ on $\mathrm{s}_{0}$

The coolant exit face $S$ is also at a specified constant pressure

$\mathrm{p}=\mathrm{p}_{\mathrm{s}}=$ constant $\mathrm{x}, \mathrm{y}$ on $\mathrm{s}$

Similarly the inlet and outlet of the jet are at constant pressure.

Equations and Boundary Conditions in Dimensionless Form

Let $F$ be the volume flow rate, per unit depth normal to the $x, y$ plane, through the porous medium or in one of the jets. For the porous medium $F$ is

found in the analysis in terms of the pressure difference $p_{0}-p_{s}$, while for the jet it is specified as $2 \mathrm{bv}_{\infty}$ (fig. 3). A characteristic velocity is $\mathrm{F} / 2 \mathrm{~b}$ as $2 \mathrm{~b}$ is used as a reference length in Figs, 1 and 2 , Using dimensionless variables

Eqs. (1), (2), and (4) become

$\tilde{\nabla} \cdot \vec{U}=0$

$\tilde{\nabla}^{2} \mathrm{~T}-\mathrm{PeU} \cdot \tilde{\nabla} \mathrm{T}=0$

$\overrightarrow{\mathrm{U}}=\tilde{\nabla} \oplus$

From the definitions of $\phi$ and $\Phi$, conditions (5) and (6) become

$\Phi=0 \quad X, Y$ on $S_{0}$

$\Phi=\Phi_{S}=$ constant $\mathrm{X}, \mathrm{Y}$ on $\mathrm{S}$

Transformation into Potential and Stream Function Coordinates

Conditions (10) and (11) show that the inlet and outlet faces of the porous media are at constant potential. From Eqs. (7) and (9)

$\tilde{\nabla} \cdot \vec{U}=\tilde{\nabla} \cdot \tilde{\nabla}=\tilde{\nabla}_{\Phi}=0$

so that in the flow regions the potential satisfies Laplace's equation. Orthogonal to the lines 

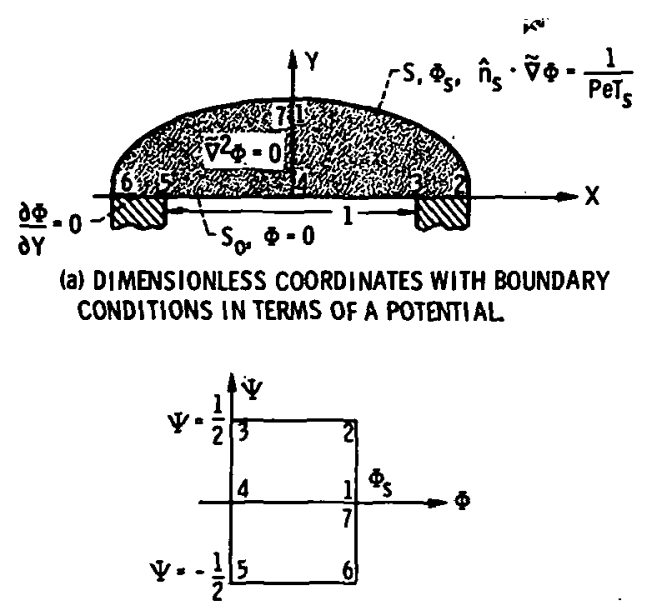

(b) REGION IN POTENTIAL PLANE

Fig. 4 POROUS IUEADING-EDGE REGION WITH COOLANTI SUPFLIED THEOUGH SLOT

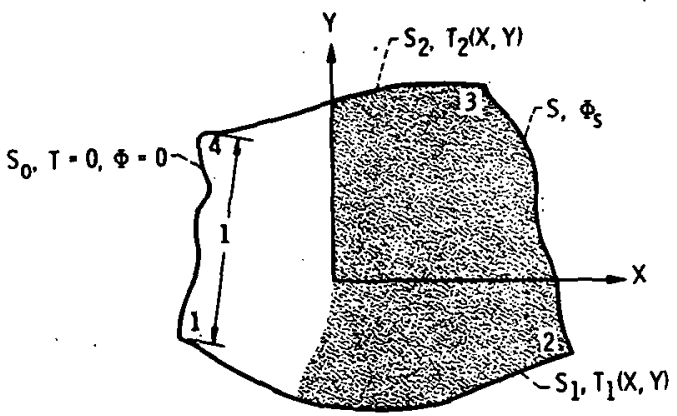

(a) REGION AND POTENTIAL BOUNDARY CONDITIONS IN DIMENSIONLESS PHYSICAL PLANE.

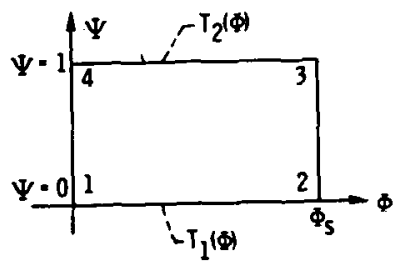

(D) REGION IN POTENTIAL PLANE.

FIg. 5 POROUS BED WITH SPECIFIED WALC TEMPERATURE VARTATIONS

are streamlines that satisfy

$\nabla^{2} \Psi=0$

The impervious boundaries $\widehat{23}$ and $\widehat{56}$ in Fig. 4(a), and $S_{1}$ and $S_{2}$ in Fig. $5(8)$ are streamines.

For the jet in Fig. 6(a) 12 and 45 are free streamlines, and the flow must be along the line of symmetry and the plate so 6789 is along a streamline. The dividing streamline 38 terminates at the stagnation point. These conditions cause the flow reg- ions to map into simple rectangles and strips in potential-stream function coordinates in Figs. 4(b), $5(b)$, and $6(b)$. This geometric simplification as well as simplifications in the differential equation and boundary conditions make it convenient to

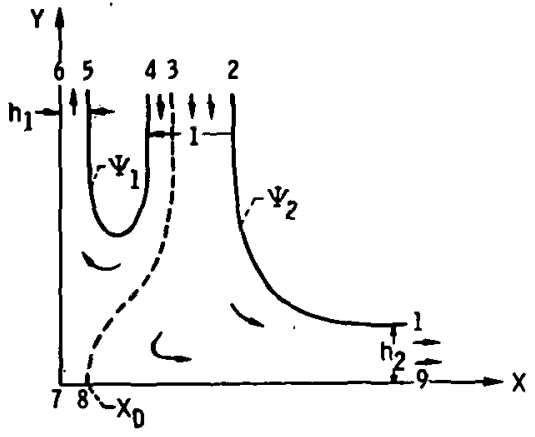

(a) DIMENSIONLESS PHYSICAL PLANE

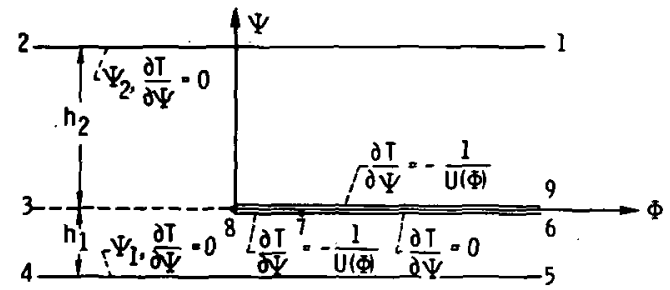

(b) FLOW REGION IN POTENTIAL PLANE

Fig. 6 IMPINGING LIQUID METAL SLOT JETS

obtain solutions in the potential plane and then conformally map the results into physical coordinates.

The pressure boundary conditions are in terms of the potential in Eqs. (10) and (11). To express the energy equation in terms of potential coordinates, Eq. ( $\vec{\theta}$ ) is used to eliminate $\vec{U}$ from $\mathrm{Eq}$. (8). The resulting equation has the same form as Eq. (16) of [1], and in [1] the details are given of the transformation into the potential plane. Using Eq. (26) of [1] yields the energy equation,

$\frac{\partial^{2} T}{\partial \Phi^{2}}-\frac{\partial^{2} T}{\partial \Psi^{2}}-P e \frac{\partial T}{\partial \Phi}=0$

This is the same equation as for convective heat transfer in a parallel plate channel with uniform flow in the direction and with the channel width in the $\Psi$ direction.

From the definition of the stream function the difference between the upper and lower streamines in Figs. $4(b), 5(b)$, and $6(b)$ is the volume flow rate between them. Since $\Psi=\psi / F$, the range of $\Psi$ is unity. When the geometries in Figs. 4 and 5 are mapped into the potential plane the range in $\Phi$ is 0 to $\Phi_{S}$. Thus the detemination of $\Phi_{S}$ from the mapping yields the volume flow rate,

$$
F=\frac{\Phi_{S}}{\Phi_{S}}=\frac{1}{\Phi_{S}}\left(p_{0}-p_{S}\right) \frac{\kappa}{\mu}
$$


Leading Edge Region with Unknown Surface Shape

In Fig. I a porous leading edge region is held by two insulated supports. The upper surface is subjected to a uniform heat flux and it is desired to maintain this surface at a uniform temperature $t_{s}$ set by design considerations. By not having any portion of the surface below $t_{s}$, the surface is not locally over cooled and coolant is not wasted. Thus the boundary conditions along $S$ are both uniform heat flux and uniform temperature, and the shape of $S$ is to be determined. Along the inlet face $S_{0}$ conservation of energy requires that any energy conducted out of the porous medium be acquired by the incoming fluid. Sumarizing these conditions :

$\left.\begin{array}{l}k_{r_{B}} \hat{n}_{s} \cdot \nabla t=q_{s}=\text { constant } \\ t=t_{s}=\text { constant }\end{array}\right\} x, y$ on $s$

$k_{\mathrm{m}} \hat{n}_{0} \cdot \nabla t=\rho C_{p}\left(t_{0}-t_{\infty}\right) \hat{n}_{0} \cdot \vec{u} \quad x, \dot{y}$ on $s_{0}$

In dimensionless form these become

$\hat{\mathrm{n}}_{\mathrm{s}} \cdot \tilde{\nabla} \mathrm{I}=1$

$\left.\begin{array}{l}\mathrm{n}_{\mathrm{s}} \cdot \overline{\nabla T}=1 \\ \mathbb{T}=\mathrm{T}_{\mathrm{S}}=\text { constant }\end{array}\right\} X, Y$ on $\mathrm{S}$

$\hat{n}_{0} \cdot \tilde{\nabla} \mathrm{I}=\mathrm{Pe} T \hat{n}_{0} \cdot \vec{U} \quad X, Y$ on $S_{O}$

Since $S_{0}$ is a line of constant potential $\hat{n}_{0}=$

$\tilde{\nabla} \cdot \tilde{\nabla}=\mathrm{Fe} T|\tilde{\nabla}|^{2} \quad X, Y$ on $S_{O}$

This problem can be reduced to a heat conduction solution which will yield the shape of $S$. The surface $S$ has potential and temperature both uni-. form. This suggests trying a solution where $T$ is only a function of $\phi$. Then Eq. (14) becomes

$\left(d^{2} T / d \Phi^{2}\right)-P e(d T / \partial \Phi)=0$

Integrating gives

$(\mathrm{dT} / \mathrm{d} \Phi)-\mathrm{Pe} \mathrm{T}=\mathrm{C}_{1}$

To obtain $\mathrm{C}_{1}$ apply the boundary condition (20) at $s_{0}$ where $\Phi=0$. Using $\tilde{\nabla}=\tilde{\nabla} \Phi(d T / d \Phi)$ reduces

$(\mathrm{dT} / \mathrm{d} \Phi)_{\left.\right|_{\Phi=0}}=\mathrm{Pe} \mathrm{T}_{\Phi=0}$

Applying Eq. (23) to Eq. (22) gives $C_{1}=0$. Then by integrating Eq. (22) from $\Phi$ to $\Phi_{s}$,

$T(\phi)=T_{S} e^{-P e\left(\Phi_{s}-\Phi\right)}=T_{S} e^{-\left[\left(p_{0}-p_{s}\right) / \alpha\right](k / \mu)}$

There still remains to satisfy the uniform heat flux along $\mathrm{S}$, condition (18a). Using $\tilde{\nabla} T=$ the condition for $\Phi$, (22) $\left.\frac{\mathrm{dT}}{\mathrm{d} \Phi}\right|_{\mathrm{S}}=\mathrm{Pe} \mathrm{T}_{\mathrm{S}}$, yields $\hat{n}_{\varepsilon} \cdot \tilde{\nabla}_{Q}=1 /\left(\mathrm{PeT}_{\mathrm{S}}\right) \quad X, Y$ on $S$

The boundary conditions for $\Phi$ are sumnarized in Fig. 4 (a) and from Eq. (12) $\Phi$ is a solution to Laplace's equation. The shape of surface $S$ has to be determined such that $\Phi$ will be constant and have a constant normal derivative along $S$. As showm in [4] and [5] this type of heat conduction problem can be solved by conformal mapping by using an auxiliary potential derivative plane. The freezing problem [4] depends on the same boundary value problem as the present case. The conformal transformations are given in [4] for mapping Fig. 4(b) into $4(\mathrm{a})$; to apply these results here requires only a change in notation. Results are given in Fig. 7 .
Two-Dimensional Heat Transfer in Porous Bed

When the porous bed in Fig. $5(a)$ is mapped into Fig. 5(b) the problem becomes convective heat transfer to uniform flow in a parallel-plate channel of unit width. Assuming the conformal mapping is known between Figs. 5(a) and $5(b)$, the $T(x, y)$ along $S_{1}$ and $s_{2}$ are transformed to yield the boundary conditions in the potential plane

$T_{1}(\Phi) \Phi, \Psi$ on $\hat{12}$

$\mathrm{T}_{2}(\Phi) \Phi, \Psi$ on $\widehat{34}$

For convective heat transfer in a channel the axial conduction term can be neglected unless $\mathrm{Pe}$ is less than about 10 so for most situations Eq. (14) can be epproximated as

$\left(\partial^{2} \mathrm{~T} / \partial \Psi^{2}\right)-\operatorname{Pe}(\partial \mathrm{T} / \partial \Phi)=0$

Without axial conduction the fluid and solid at the entrance of the bed are at the reservoir temperature,

$T=0 \quad \Phi, \Psi$ on 14

Equation (27) has the same form as the one dimensional transient conduction equation. The solution in the potential plane is found by using results for a slab of constant thickness initially at zero temperature and then heated in a time dependent manner as given by conditions (26). From Eq. ( 1 ) on page 103 of [6] the temperature distribution is given by

$$
\begin{aligned}
& T(\Phi, \Psi)=\frac{1}{P e} \sum_{n=1}^{\infty} n e^{-\left(n^{2} / P e\right) \pi^{2} \Phi} \sin n \pi \Psi \\
& \int_{0}^{\Phi} e^{\left(n^{2} / P e\right) \pi^{2} \lambda}\left[T_{1}(\lambda)-(-1)^{n_{T}} T_{2}(\lambda)\right] d \lambda
\end{aligned}
$$

Since there is the same volume flow in each $d \Psi$ layer, the average exit temperature from the bed is $\overline{\mathrm{T}}\left(\Phi_{\mathrm{S}}\right)=\int_{0}^{1} \mathrm{~T}\left(\Phi_{\mathrm{S}} ; \Psi\right) \mathrm{d} \Psi$

The total heat transferred by the bed to the fluid is $Q_{\text {tot }}=\rho C_{p} F\left(\bar{t}_{s}-t_{\infty}\right)=\rho C_{p} F\left(t_{w, r}-t_{\infty}\right) \bar{T}\left(\Phi_{s}\right)$. Inserting $T\left(\Phi_{S}, \Psi\right)$ from $E q$. (29) and integrating to obtain $\overline{\mathrm{T}}\left(\Phi_{\mathrm{S}}\right)$ yield

$$
\begin{gathered}
\frac{Q_{t o t}}{\rho C_{p} F\left(t_{w, r}-t_{\infty}\right)}=\frac{4}{\pi \mathrm{Fe}} \sum_{n=1,3,5 \ldots}^{\infty} e^{-\left(n^{2} / P e\right) \pi^{2} \Phi_{s}} \\
\int_{0}^{\Phi_{s}} e^{\left(n^{2} \pi^{2} / \mathrm{Pe}\right) \lambda}\left[\mathrm{T}_{1}(\lambda)-(-1)^{\left.\mathrm{n}_{\mathrm{T}}(\lambda)\right] d \lambda}\right.
\end{gathered}
$$

The $F$ is obtained from Eq. (15) by using the $\Phi_{s}$ found in the mapping.

\section{Heat Transfer for Two Impinging Jets}

As given in [7] the impinging jet in Fig. 6(a) maps into the potential region in Fig. 6(b); this represents a uniform flow in the $\Phi$ direction. With axial conduction neglected the heat transfer can be computed as convection to two separate flows in channels $h_{1}$ and $h_{2}$ wide. The heat losses along the free streamlines are neglected. The normals to the free streamlines are along the $\mathbf{\Psi}$ direction so that

$(\partial \mathrm{T} / \partial \Psi)=0 \quad 0, \Psi$ on $\widehat{12}$ and $\overparen{45}$ 
From symetry about streamline 67

$$
(\partial \mathrm{T} / \partial \Psi)=0 \quad \Phi, \Psi \text { on } 67
$$

The plate $\overparen{789}$ is heated with a uniform flux $q_{W}=$ $-\mathrm{k} \partial \mathrm{t} / \partial \mathrm{y} \mid \mathrm{y}=0$ or

$$
(\partial \mathrm{T} / \partial \mathrm{Y})=-1 \quad \mathrm{X}, \mathrm{Y} \text { on } \widehat{789}
$$

This must be transformed into the potential plane. Using $\partial \mathrm{T} / \partial \mathrm{Y}=(\partial \mathrm{T} / \partial \Psi)(\partial \Psi / \partial \mathrm{Y})+(\partial \mathrm{T} / \partial \Phi)(\partial \Phi / \partial \mathrm{Y})$ and the relations along 789 that $\Psi=0$ and $\partial_{\Phi} / \partial Y=0$ (since $\mathrm{v}=0$ ) yield

$-1=\left.\left.(\partial T / \partial \Psi)\right|_{\Psi=0}(\partial \Psi / \partial Y)\right|_{Y=0}$

From Eq. (9) and the Cauchy-Riemann equations, $U=$ $\partial \phi / \partial X=\partial \Psi / \partial Y$. Condition (35) becomes

$$
(\partial \mathrm{T} / \partial \Psi)=-1 / \mathrm{U}(\Phi) \quad \Phi, \Psi \text { on } \overparen{789}
$$

The boundary conditions are sumarized in Fig. $6(\mathrm{~b})$. The velocity $U(\Phi)$ along 789 needed in Eq. (36) is given by the conformal mapping solution for impinging jets in [7].

With these boundary conditions the channels of width $h_{1}$ and $h_{2}$ have nonuniform heat addition along 78 and $\widehat{89}$, and the other boundaries are insulated: since Eq. (27) has the same form as the transient conduction equation, the condition (36) can be thought of as a heat flux variation with time. In [6] the transient temperature solution is given for suddenly imposed heating at one surface of a slab with the other surface insulated. The temperature variation at the heated surface for an imposed flux of unit magnitude is $G(\Phi)$. By superposition, for a variable heat flux,

$\mathrm{T}_{\mathrm{W}}(\Phi)=\int_{0}^{\Phi} \frac{1}{U(\Phi-\tau)} \frac{\mathrm{dG}}{d \tau} d \tau$

Differentiating the $G(\Phi)$ given in [6], page 112 , the temperature distribution along the heated plate becames,

$$
\begin{gathered}
\mathrm{T}_{\mathrm{w}}(\Phi)=\frac{\left(\mathrm{t}_{\mathrm{w}}-\mathrm{t}_{\infty}\right) \mathrm{k}}{2 b \mathrm{q}_{\mathrm{w}}}=\left(\frac{1}{\pi \mathrm{Pe}}\right)^{1 / 2} \int_{0}^{\Phi} \frac{1}{\tau^{1 / 2}|\mathrm{U}(\Phi-\tau)|} \\
\sum_{\mathrm{m}=0}^{\infty}\left[\mathrm{e}^{-\left(\mathrm{Pem}^{2} \mathrm{~h}_{\mathrm{n}}^{2}\right) / \tau}+\mathrm{e}^{\left.-\left[\mathrm{Pe}(\mathrm{m}+1)^{2} \mathrm{~h}_{\mathrm{n}}^{2}\right] / \tau\right]} d \tau\right.
\end{gathered}
$$

where $h_{n}=h_{1}$ for $0 \leq x \leq x_{D}$ and $h_{n}=h_{0}$ for $\mathrm{x}_{\mathrm{D}} \leq \mathrm{X} \leq \infty$, and $\mathrm{h}_{7}$ and $\mathrm{h}_{2}$ are in [7]. The mapping relations in [7] are used to transform $T_{W}(\Phi)$ to $\mathrm{T}_{\mathrm{W}}(\mathrm{X})$. Typical temperature distributions are in Fig. 8.

\section{DISCUSSION}

The shapes of a porous cooled region are shown in Fig. $7(a)$. that simultaneously satisfy the conditions of uniform heat flux and temperature along the coolant exit face. The governing parameter involves the overall temperature and pressure differences, and the surface heat flux. If the allowable surface temperature $t_{s}$ is increased, less coolant flow is required. The region thickness is increased thereby reducing the flow since the pressure difference is fixed. If the surface flux $q_{s}$ is increased, a greater flow is required which corresponds to a thinner region. The coolant flow is given in Fig. 7 (b).

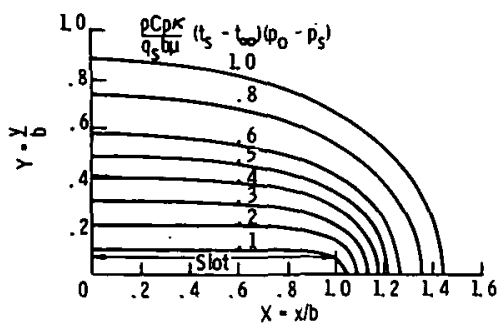

(a) SHAPES OF REGION FOR VARIOUS VALUES OF IMPOSED PHYSICAL CONDITIONS.

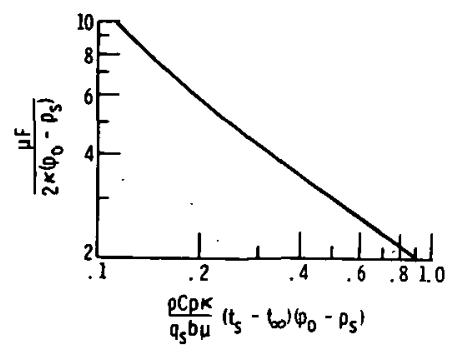

(b) COOLANT VOLUME FLOW AS A FUNCTION OF IMPOSED PHYSICAL CONDITIONS.

FIG. 7. - POROUS LEADING EDGE REGION.

F1gure 8 shows the temperature distribution along a uniformly heated plate for two impinging slot jets (from symmetry only half the geometry is shown). The surface temperature goes toward infinity as the velocity approaches zero in the backflow region at $x=0$; this is a consequence of neglecting heat conduction along the streamlines (see Fig. 8 of [3]).

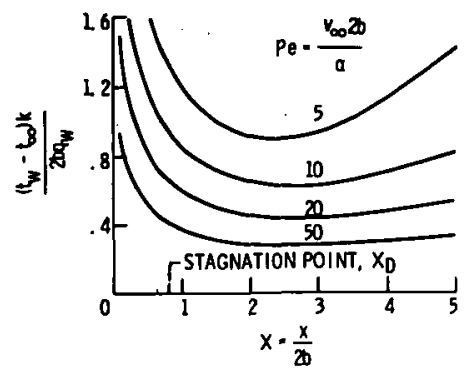

Fig. 8 DIMENSIONLESS TEMPERATURE ALONG HEATED WALL FOR IMPINGING PARALUEL LIQUID METAL SLOT JETS, $S / \mathrm{b}=3$.

\section{REFERENCES}

[i] Siegel, R. and M. E. Goldstein: NASA TN D-5878 (1970).

[2] Siegel, R. and M. E. Goldstein: NASA TN D-694] (1972).

[3] Grosh, R. J. and R. D. Cess: Trans. ASME 80, 667 (1958).

[4] Siegel, R.: NASA TN D-4771 (1968).

[5] Goldstein, M. E. and R. Siegel: Int. J. Heat and Mass Transfer, 13, i632 (1970).

[6] Carslaw, H. S. and J. C. Jaeger: "Conduction of Heat in Solids," 2nd ed., Oxford Univ. Press (1959).

[7] Gedney, R. T. and R. Siegel: NASA TN D-4957 (1968). 